\title{
Avaliação do autoconhecimento da equipe médica, de enfermagem e farmácia sobre medicamentos potencialmente perigosos
}

\section{Self-knowledge assessment of the nursing and pharmacy medical teams on potentially hazardous drugs}

Antonia Amanda Lima Souza ${ }^{1}$. Eugenie Desirée Rabelo Néri² ${ }^{2}$ Glauciene Cardoso Gomes ${ }^{1}$. Emeline Moura Lopes $^{3}$. Marta Maria de França Fonteles ${ }^{4}$. Assuero Silva Meira ${ }^{5}$. Hemerson Bruno da Silva Vasconcelos ${ }^{6}$.

1 Especialista em Saúde da Mulher e da Criança, Programa de Residência Multiprofissional em Saúde da Mulher e da Criança, Universidade Federal do Ceará (UFC), Fortaleza, Ceará, Brasil. 2 Mestre em Ciências Farmacêuticas, Maternidade Escola Assis Chateaubriand (MEAC), Fortaleza, Ceará, Brasil. 3 Doutora em Enfermagem na Promoção da Saúde, Maternidade Escola Assis Chateaubriand (MEAC), Fortaleza, Ceará, Brasil. 4 Doutora em Farmacologia, Universidade Federal do Ceará (UFC), Fortaleza, Ceará, Brasil. 5 Doutor em Farmacologia, Maternidade Escola Assis Chateaubriand (MEAC), Fortaleza, Ceará, Brasil. 6 Graduação em Estatística, Maternidade Escola Assis Chateaubriand (MEAC), Fortaleza, Ceará, Brasil.

\section{RESUMO}

Objetivo: determinar o grau de conhecimento sobre medicamentos potencialmente perigosos (MPP), entre médicos, equipe de enfermagem, equipe de farmácia e residentes. Método: estudo transversal e quantitativo realizado com 157 profissionais pertencentes à equipe médica, farmacêutica, de enfermagem e residentes. O estudo foi realizado em duas fases: I) avaliação do conhecimento e; II) intervenção educativa. Foram utilizadas escalas de avaliação da opinião (Likert) e de avaliação do conhecimento. Resultados: na fase I foram aplicados 157 questionários, identificando que 88,5\% (n=139) dos profissionais não havia participado de treinamentos sobre MPP nos últimos seis meses, e 47,1\% ( $n=74)$ declarou conhecimento razoável sobre MPP. Foi identificada diferença significativa entre as categorias profissionais quanto ao conhecimento geral declarado sobre MPP (Quiquadrado; $\mathrm{p}<0,001$ ) e sobre os medicamentos da lista de MPP da instituição (Qui-quadrado; $\mathrm{p}=0,001$ ). O relato de ter presenciado erros envolvendo MPP foi mais frequente entre profissionais com maior tempo de serviço (Qui-quadrado; p=0,006). A fase II envolveu 164 profissionais, 90 deles participantes da Fase I. Conclusão: foi evidenciada fragilidade no conhecimento sobre MPP. Sugere-se sensibilizar os profissionais para as consequências danosas dos erros envolvendo MPP e a adoção de ferramentas de suporte (listas e protocolos).

Palavras-chave: Lista de medicamentos potencialmente inapropriados. Conhecimento. Segurança do paciente.

\begin{abstract}
Objective: to identify the knowledge level on potentially hazardous drugs (PHD) among doctors, the nursing and pharmacy team and residents. Methodology: this is a transversal, quantitative study developed with 157 professionals belonging to nursing, pharmacy, medical teams and residents. The study was developed in two stages: I) knowledge assessment and II) educational intervention. Opinion (Likert) and knowledge assessment scales were applied. Results: in phase I, 157 questionnaires were identified, being that $88.5 \%(\mathrm{n}=139)$ of professionals had not participated in PHD trainings during the last six months whereas $47.1 \%(n=74)$ declared to have a reasonable knowledge on MPP. It was identified a significant difference between the professional categories with regards to their general knowledge on PHD (chi-square; $p<0.001$ ) and on the institution's PHD list (Chi-square$\mathrm{p}=0.001$ ). The narration of having witnessed errors involving PHD was more frequent among professionals with longer service time (Chi-square; $p=0.006$ ). Stage II involved 164 professionals, out of which 90 also took part in Stage I. Conclusion: there was evidence of fragility in the knowledge on PHD. We suggest raising awareness among professionals on the harmful consequences of PHD, thus becoming necessary to include support tools such as lists and protocols.
\end{abstract}

Keywords: List of potentially inadequate drugs. Knowledge. Patient safety.

Autor correspondente: Antonia Amanda Lima Souza, Rua Coronel Nunes de Melo, sem número, Rodolfo Teófilo, Fortaleza, Ceará. CEP: 60430270. Telefone: +5585 98751-9144. E-mail: amandalima596@gmail.com

Conflito de interesses: Não há qualquer conflito de interesses por parte de qualquer um dos autores.

Recebido em: 21 Fev 2018; Revisado em: 24 Mai 2018; Aceito em: 19 Jul 2018. 


\section{INTRODUÇÃO}

A Organização Mundial da Saúde (OMS) criou, em 2004, a Aliança Mundial para a Segurança do paciente, que objetivou contribuir para a redução ao mínimo aceitável dos danos associados ao cuidado de saúde, neles envolvidos os riscos do uso dos medicamentos. ${ }^{1,2}$

Considerando que para prevenir os erros de medicação, sobretudo aqueles de maior gravidade, é necessário implementar medidas que reduzam a possibilidade de que os erros aconteçam. Uma importante medida é a aplicação do Protocolo de segurança na prescrição, uso e administração de medicamentos, ${ }^{3}$ com foco especial no manejo dos medicamentos potencialmente perigosos (MPP). Certos erros de medicação não ocasionam danos aos pacientes, porém, caso envolva MPP os danos podem ser graves. ${ }^{4}$

Medicamentos Potencialmente Perigosos são os medicamentos que quando envolvidos em erros, a gravidade do dano é maior que as observadas com os demais medicamentos e, quando envolvidos em falhas, resultam, em consequências danosas para o paciente. ${ }^{5}$ Em estudo realizado por Rosa e colaboradores foi identificado que de 7.148 MPP prescritos, 3.177 prescrições apresentavam algum tipo de erro. ${ }^{6}$

Nos estabelecimentos de saúde é comum a administração de MPP aos pacientes internados, sendo necessário maior atenção por parte dos profissionais de saúde envolvidos, para que esse processo seja seguro para o paciente. Neste sentido, o Institute for Safe Medication Practices (ISMP), instituição não governamental voltada para uso seguro de medicamentos, e por meio do ISMP-Brasil, divulga regularmente a lista de MPP e recomenda a adoção de estratégias para reduzir erros de medicação. ${ }^{7}$

Ações ligadas à padronização dos MPP, limitando as formas farmacêuticas e as concentrações; estabelecendo quantidades desses medicamentos nas unidades hospitalares; realização de dupla checagem na dispensação e administração, têm sido sugeridas para prevenir erros e danos graves relacionados à estes medicamentos. ${ }^{8}$ Porém, de nada adianta tamanho esforço se os profissionais não forem capazes de perceber os riscos envolvidos, se não identificarem quais são os MPP e a importância das ações preventivas, pois erros de medicação acontecem por vários motivos, dentre eles, a deficiência de conhecimento do profissional sobre o medicamento. ${ }^{9}$

Diversas pesquisas têm abordado o conhecimento e capacitação dos profissionais de saúde para a identificação e o manejo dos MPP. Pesquisa randomizada realizada em Taiwan, envolvendo 232 enfermeiros, identificou que $74,1 \%$ deles não tinham nenhum treinamento sobre MPP. ${ }^{10}$ Outro estudo no mesmo país verificou que apenas $16,7 \%$ dos participantes tinham treinamento sobre MPP e 84,6 \% relataram a necessidade de treinamento adicional sobre estes medicamentos. ${ }^{11}$ Estes achados foram complementados por Lo e colaboradores, que concluiu ser essencial fornecer conhecimentos sobre MPP na educação acadêmica e na continuada aos profissionais de saúde. ${ }^{12}$ Por sua vez, um estudo conduzido no Hospital das
Clínicas de Ribeirão Preto, apresentou que apenas 26,7\% dos enfermeiros tinham treinamento sobre MPP. ${ }^{13}$

Diante do exposto, determinar o grau de conhecimento das equipes médica, de enfermagem e de farmácia de uma maternidade do estado do Ceará sobre os MPP, constitui-se em uma importante estratégia para mapear deficiências e planejar intervenções educativas ajustadas às necessidades institucionais.

\section{MÉTODOS}

Estudo transversal e quantitativo realizado no período de agosto a novembro de 2016, em uma Maternidade Escola do nordeste Brasileiro. Participaram da pesquisa médicos, enfermeiros, auxiliares e técnicos de enfermagem; farmacêuticos e técnicos de farmácia; residentes médicos e multiprofissionais. Foram excluídos do estudo os profissionais que estavam afastados do ambiente de trabalho, por licença médica e maternidade, férias e os que não aceitaram participar de todas as fases da pesquisa.

O tamanho amostral calculado foi de $n=149$, considerou um erro amostral de 5\%, nível de confiança de $90 \%$ e percentual máximo de $80 \%$ de conhecimento sobre MPP entre os participantes. ${ }^{10}$ Os profissionais foram selecionados aleatoriamente, por conglomerado, utilizando-se as escalas de trabalho e tábua de números aleatórios. ${ }^{14}$

O estudo foi realizado em duas fases, uma dedicada à aplicação do questionário para avaliação do conhecimento (Fase I) e outra a elaboração e aplicação da intervenção educativa (Fase II).

\section{Fase I: aplicação de questionário para avaliação do conhecimento sobre MPP}

Foi aplicado um questionário eletrônico, estruturado, elaborado na plataforma Google Docs ${ }^{\circledR}$, composto por 19 perguntas.

A escala de Likert ${ }^{15}$ foi pontuada com os seguintes escores: "concordo totalmente/suficiente"(100), "concordo parcialmente/relativamente suficiente"(75), "não concordo, nem discordo/razoável"(50), “discordo/insuficiente"(25) e "discordo totalmente/extremamente insuficiente"(0) para medir o nível de concordância de cada item. De acordo com o nível de concordância assinalado, foi calculada a média dos escores em cada questão. Para interpretação das médias encontradas, foi utilizada a seguinte escala de conhecimento, elaborada pelos autores: $<50$ escores $=$ conhecimento escasso; 50 a 74 escores $=$ conhecimento em desenvolvimento e $\geq 75$ escores $=$ conhecimento estabelecido.

O conhecimento do participante foi avaliado como se segue:

1)Auto avaliação do conhecimento sobre MPP: foi calculada mediante a atribuição do escore à resposta do participante para a questão 12, segundo escala de Likert, seguido da aplicação da escala de conhecimento. 
2) Conhecimento geral declarado sobre MPP: foi calculado pela aplicação da escala de conhecimento sobre a média do valor dos escores para as questões de 11 a 16, de cada participante. Esta variável avalia a extensão do conhecimento sobre os processos que envolvem os MPP.

3) Conhecimento real demonstrado sobre MPP: essa variável representa o percentual de acerto do participante em identificar os MPP em uma lista contendo MPP e outros medicamentos (pergunta 17) e, após a aplicação da escala de conhecimento, indica o real conhecimento sobre a lista de MPP demonstrado pelo participante.

Os questionários foram aplicados com auxílio de tablet conectado à internet ou com o questionário impresso.

\section{Fase II: elaboração e aplicação da intervenção educativa:}

Tomando como referência os resultados obtidos na Fase I, foi elaborada intervenção educativa sobre MPP, contemplando as principais lacunas de conhecimento identificadas. O treinamento foi planejado utilizando metodologia da aprendizagem significativa baseada na Andragogia. ${ }^{16}$

O objetivo da intervenção era informar sobre o que são MPP, quais MPP estão padronizados na instituição, como identificar um MPP na instituição, cuidados na prescrição, dispensação e administração, além de outros pontos identificados como lacunas de conhecimento, após análise da Fase I.

A intervenção educativa foi realizada pela pesquisadora principal, com apoio da equipe do setor de Vigilância em
Saúde e Segurança do Paciente da instituição, sendo utilizado como recurso audiovisual para apresentação slides em Power Point ${ }^{\circledR}$ e álbum seriado (utilizado em locais sem acesso a computador). Como material de apoio foi utilizado folder informativo, elaborado a partir das dúvidas referidas na Fase I. O tempo médio dos treinamentos foi de 15 minutos, sendo os mesmos realizados nos locais de trabalho dos profissionais participantes.

Os dados coletados foram analisados utilizando-se planilha do programa Microsoft Office Excel $^{\mathbb{R}}$ versão 2013, e o programa Statistical Package for the Social Sciences (SPSS) versão 22.0. Os dados foram analisados utilizando-se estatística descritiva e foram utilizados os testes Qui-quadrado de Pearson e teste de correlação Gama.

O presente estudo foi previamente aprovado pelo Comitê de Ética em Pesquisa da instituição e recebeu o CAAE 55908416.5.0000.5050.

\section{RESULTADOS}

$\mathrm{Na}$ Fase I do estudo responderam ao questionário 157 profissionais de saúde. O quantitativo de participantes foi superior ao mínimo definido para: farmacêuticos $(600 \%$; $\mathrm{n}=12)$, residentes multiprofissionais $(275 \% ; \mathrm{n}=11)$ e técnicos de farmácia $(600 \% ; n=12)$, e inferior para médicos $(44,8 \%$; $\mathrm{n}=13)$ e médicos residentes $(40 \% ; \mathrm{n}=2)$.

O perfil dos profissionais que responderam o questionário de conhecimento sobre MPP está apresentado na Tabela 1.

Tabela 1. Perfil dos profissionais de saúde participantes da pesquisa sobre conhecimento de medicamentos potencialmente perigosos em Maternidade de referência do nordeste Brasileiro, Brasil, 2016.

\begin{tabular}{|c|c|c|c|c|c|c|c|c|}
\hline $\begin{array}{l}\text { Profissionais } \\
\text { participantes }\end{array}$ & Enfermeiro & Farmacêutico & Médico & $\begin{array}{c}\text { Auxiliar e } \\
\text { Técnico de } \\
\text { enfermagem }\end{array}$ & $\begin{array}{l}\text { Técnico } \\
\text { de } \\
\text { farmácia }\end{array}$ & $\begin{array}{l}\text { Médico } \\
\text { residente. }\end{array}$ & $\begin{array}{c}\text { Residente } \\
\text { multiprofissional. }\end{array}$ & Total \\
\hline & $\mathrm{n}(\%)$ & $\mathrm{n}(\%)$ & $\mathrm{n}(\%)$ & $\mathrm{n}(\%)$ & $\mathrm{n}(\%)$ & $\mathrm{n}(\%)$ & $\mathrm{n}(\%)$ & $\mathrm{n}(\%)$ \\
\hline Participantes & $32(20,4)$ & $12(7,6)$ & $13(8,3)$ & $75(47,8)$ & $12(7,6)$ & $2(1,3)$ & $11(7,0)$ & $157(100)$ \\
\hline \multicolumn{9}{|l|}{ Sexo } \\
\hline Masculino & $1(3,1)$ & $3(25,0)$ & $8(61,5)$ & $4(5,3)$ & $3(25,0)$ & $0(0,0)$ & $2(18,2)$ & $21(13,4)$ \\
\hline Feminino & $31(96,9)$ & $9(75,0)$ & $5(38,5)$ & $71(94,7)$ & $9(75,0)$ & $2(100,0)$ & $9(81,8)$ & $136(86,6)$ \\
\hline \multicolumn{9}{|l|}{ Faixa etária } \\
\hline 18 a 25 anos & $1(3,1)$ & $0(0,0)$ & $0(0,0)$ & $8(10,7)$ & $1(8,3)$ & $0(0,0)$ & $4(36,4)$ & $14(8,9)$ \\
\hline 26 a 30 anos & $7(21,9)$ & $2(16,7)$ & $0(0,0)$ & $14(18,7)$ & $2(16,7)$ & $1(50,0)$ & $5(45,5)$ & $31(19,7)$ \\
\hline 31 a 39 anos & $21(65,6)$ & $8(66,7)$ & $3(23,1)$ & $32(42,7)$ & $7(58,3)$ & $1(50,0)$ & $0(0,0)$ & $72(45,9)$ \\
\hline 40 a 49 anos & $2(6,3)$ & $2(16,7)$ & $7(53,8)$ & $16(21,3)$ & $2(16,7)$ & $0(0,0)$ & $2(18,1)$ & $31(19,7)$ \\
\hline 50 a 59 anos & $1(3,1)$ & $0(0,0)$ & $3(23,1)$ & $4(5,3)$ & $0(0,0)$ & $0(0,0)$ & $0(0,0)$ & $8(5,1)$ \\
\hline$>60$ anos & $0(0,0)$ & $0(0,0)$ & $0(0,0)$ & $1(1,3)$ & $0(0,0)$ & $0(0,0)$ & $0(0,00)$ & $1(0,6)$ \\
\hline \multicolumn{9}{|l|}{ Pós-graduação } \\
\hline Sim & $30(93,8)$ & $12(100)$ & $13(100)$ & $15(20,0)$ & $2(16,7)$ & $0(0,0)$ & $2(18,2)$ & $74(47,1)$ \\
\hline Não & $2(6,3)$ & $0(0,0)$ & $0(0,0)$ & $60(80,0)$ & $10(83,3)$ & $2(100)$ & $9(81,8)$ & $83(52,9)$ \\
\hline
\end{tabular}

Continua. 
Conclusão.

Tabela 1. Perfil dos profissionais de saúde participantes da pesquisa sobre conhecimento de medicamentos potencialmente perigosos em Maternidade de referência do Nordeste Brasileiro, Brasil, 2016.

\begin{tabular}{|c|c|c|c|c|c|c|c|c|}
\hline $\begin{array}{l}\text { Profissionais } \\
\text { participantes }\end{array}$ & Enfermeiro & Farmacêutico & Médico & $\begin{array}{l}\text { Auxiliar e } \\
\text { Técnico de } \\
\text { enfermagem }\end{array}$ & $\begin{array}{l}\text { Técnico } \\
\text { de } \\
\text { farmácia }\end{array}$ & $\begin{array}{l}\text { Médico } \\
\text { residente. }\end{array}$ & $\begin{array}{c}\text { Residente } \\
\text { multiprofissional. }\end{array}$ & Total \\
\hline & n $(\%)$ & n $(\%)$ & n $(\%)$ & n $(\%)$ & n $(\%)$ & n $(\%)$ & n $(\%)$ & n $(\%)$ \\
\hline \multicolumn{9}{|c|}{ Tempo de Profissão } \\
\hline$<1$ ano & $0(0,0)$ & $0(0,0)$ & $0(0,0)$ & $2(2,7)$ & $5(41,7)$ & $0(0,0)$ & $3(27,3)$ & $10(6,4)$ \\
\hline 1 a 2 anos & $2(6,3)$ & $0(0,0)$ & $0(0,0)$ & $7(9,3)$ & $3(25,0)$ & $0(0,0)$ & $7(63,6)$ & $19(12,1)$ \\
\hline 3 a 5 anos & $2(6,3)$ & $0(0,0)$ & $0(0,0)$ & $14(18,7)$ & $0(0,0)$ & $1(50,0)$ & $0(0,0)$ & $17(10,8)$ \\
\hline 6 a 10 anos & $16(50,0)$ & $6(50,0)$ & $3(23,1)$ & $22(29,3)$ & $3(25,0)$ & $1(50,0)$ & $0(0,0)$ & $51(32,5)$ \\
\hline 11 a 20 anos & $10(31,3)$ & $6(50,0)$ & $7(53,8)$ & $24(32,0)$ & $1(8,3)$ & $0(0,0)$ & $1(9,1)$ & $49(31,2)$ \\
\hline 21 a 29 anos & $2(6,3)$ & $0(0,0)$ & $2(15,4)$ & $6(8,0)$ & $0(0,0)$ & $0(0,0)$ & $0(0,0)$ & $10(6,4)$ \\
\hline$>30$ anos & $0(0,0)$ & $0(0,0)$ & $1(7,7)$ & $0(0,0)$ & $0(0,0)$ & $0(0,0)$ & $0(0,0)$ & $1(0,6)$ \\
\hline \multicolumn{9}{|c|}{ Tempo de trabalho na instituição } \\
\hline$<6$ meses & $12(37,5)$ & $0(0,0)$ & $0(0,0)$ & $22(29,3)$ & $1(8,3)$ & $0(0,0)$ & $4(36,4)$ & $39(24,9)$ \\
\hline 7 meses a 1 ano & $14(43,8)$ & $6(50,0)$ & $2(15,4)$ & $32(42,7)$ & $11(91,7)$ & $0(0,0)$ & $5(45,5)$ & $70(44,6)$ \\
\hline 2 a 4 anos & $2(6,3)$ & $3(25,0)$ & $3(23,1)$ & $5(6,7)$ & $0(0,0)$ & $2(100)$ & $2(18,2)$ & $17(10,8)$ \\
\hline 5 a 10 anos & $1(3,1)$ & $1(8,3)$ & $2(15,4)$ & $4(5,3)$ & $0(0,0)$ & $0(0,0)$ & $0(0,0)$ & $8(5,1)$ \\
\hline 11 a 19 anos & $2(6,3)$ & $2(16,7)$ & $3(23,1)$ & $8(10,7)$ & $0(0,0)$ & $0(0,0)$ & $0(0,0)$ & $15(9,6)$ \\
\hline 20 a 25 anos & $1(3,1)$ & $0(0,0)$ & $2(15,4)$ & $1(1,3)$ & $0(0,0)$ & $0(0,0)$ & $0(0,0)$ & $4(2,5)$ \\
\hline 26 a 29 anos & $0(0,0)$ & $0(0,0)$ & $1(7,7)$ & $3(4,0)$ & $0(0,0)$ & $0(0,0)$ & $0(0,0)$ & $4(2,5)$ \\
\hline$>30$ anos & $0(0,0)$ & $0(0,0)$ & $0(0,0)$ & $0(0,0)$ & $0(0,0)$ & $0(0,0)$ & $0(0,0)$ & $0(0,0)$ \\
\hline
\end{tabular}

Quando questionados sobre sua participação em alguma capacitação sobre MPP nos últimos seis meses, 88,5\% (n=139) relataram não ter participado de treinamentos. Dos que participaram de treinamento, 55,5\% eram auxiliar/técnicos de enfermagem $(n=10), 16,7 \%$ enfermeiros $(n=3), 16,7 \%$ farmacêuticos $(n=3)$ e $11,1 \%$ técnicos de farmácia $(n=2)$. A realização de treinamentos sobre MPP na instituição foi avaliada como essencial por $94,9 \%(n=149)$ dos participantes.

Os participantes foram solicitados a realizar a avaliação sobre o seu grau de conhecimento sobre MPP (Questão 12), sendo identificado que $3,2 \%(n=5)$ declararam ter conhecimento suficiente sobre o tema e $1,3 \%(n=2)$ extremamente insuficiente. Quanto ao conhecimento sobre os medicamentos que compõem a lista dos MPP da instituição (Questão 13), 12,1\% (n=19) dos participantes informou que conhecia estes medicamentos.

Em relação ao conhecimento sobre o armazenamento de MPP, $16,6 \%(n=26)$ dos participantes declarou conhecer integralmente o processo. A maioria dos residentes multiprofissionais $(63,6 \% ; n=7)$ desconhecem como é feito o armazenamento, por outro lado, parte dos enfermeiros (65,6\%; $\mathrm{n}=21)$, farmacêuticos $(100 \% ; \mathrm{n}=12)$, médicos $(38,5 \% ; n=5)$, os técnicos/auxiliares de enfermagem $(69,4 \% ; n=52)$ e os técnicos de farmácia $(91,7 \% ; n=11)$ declararam conhecer. Os residentes médicos apresentaram opinião dividida.

No tocante à identificação dos MPP, conheciam integralmente o processo $28,7 \%(\mathrm{n}=45)$ dos participantes. Os residentes multiprofissionais $(63,6 \% ; n=7)$ e $53,8 \%$ dos médicos $(n=7)$ desconhecem o processo, porém, os residentes médicos $50 \%$ $(n=1)$ desconhecem como é realizado a identificação dos MPP e $50 \%(n=1)$ ficaram neutros.

Quanto à checagem dos MPP, verificou-se que 23,6\% $(n=37)$ dos participantes conhecia totalmente o processo. Dentre os farmacêuticos $100 \%(n=12), 59,4 \%$ dos enfermeiros $(n=19)$, $69,3 \%$ dos técnicos/auxiliares de enfermagem $(n=52)$ e $91,6 \%$ dos técnicos de farmácia $(\mathrm{n}=11)$, informaram conhecer como é realizada a conferência dos MPP. Por outro lado, a maioria dos médicos $(61,5 \% ; n=8)$ e residentes multiprofissionais $(63,6 \%$; $\mathrm{n}=7$ ) relataram desconhecer o processo. Dos residentes médicos, $50 \%(\mathrm{n}=1)$ deles desconhece como é realizado a checagem dos MPP e $50 \%(n=1)$ ficou neutro.

O conhecimento geral declarado sobre MPP foi avaliado entre os participantes (Tabela 2), não sendo identificada variação associada ao tempo de serviço (Teste de correlação gama, $p=0,172$ ). Foi identificada diferença significativa entre as categorias profissionais quanto ao conhecimento geral declarado sobre MPP (Qui-quadrado, $\mathrm{p}<0,001)$ e quanto ao conhecimento sobre os medicamentos que compõem a lista de MPP da instituição (Qui-quadrado, $\mathrm{p}=0,001$ ). O conhecimento declarado da lista (Questão 13) não variou significativamente entre profissionais com diferentes tempos de serviço (Teste de correlação gama; $p=0,199$ ). 
Quando comparada a auto avaliação do conhecimento sobre a lista de MPP da instituição e o conhecimento real demonstrado sobre MPP, foi identificada diferença entre as categorias profissionais (Qui-quadrado, $\mathrm{p}=0,002$ ). A equipe de enfermagem foi o grupo que apresentou o maior desvio entre o conhecimento declarado e o real, no reconhecimento dos MPP (Tabela 3).
A pergunta (Questão17) era composta por 23 medicamentos dos quais $69,6 \%(n=16)$ eram MPP, segundo a lista atualizada do ISMP. ${ }^{7}$ Os MPP mais reconhecidos foram o cloreto de potássio $(88,5 \% ; n=139)$, a dobutamina $(78,3 \% ; n=123)$ e o sulfato de magnésio $(79,6 \% ; n=125)$, (Gráfico 1).

Tabela 2. Conhecimento geral declarado sobre medicamentos potencialmente perigosos, pelos profissionais participantes da pesquisa sobre conhecimento de medicamentos potencialmente perigosos em uma maternidade de referência do nordeste Brasileiro, Brasil, 2016.

\begin{tabular}{|c|c|c|c|c|c|c|}
\hline \multirow[b]{2}{*}{ Categoria profissional } & \multicolumn{2}{|c|}{$\begin{array}{l}\text { Conhecimento } \\
\text { escasso }\end{array}$} & \multicolumn{2}{|c|}{$\begin{array}{l}\text { Conhecimento em } \\
\text { desenvolvimento }\end{array}$} & \multicolumn{2}{|c|}{$\begin{array}{c}\text { Conhecimento } \\
\text { estabelecido }\end{array}$} \\
\hline & n & $\%$ & $\mathrm{n}$ & $\%$ & n & $\%$ \\
\hline Enfermeiro $(\mathrm{n}=32)$ & 06 & 18,8 & 12 & 37,5 & 14 & 43,7 \\
\hline Farmacêutico $(\mathrm{n}=12)$ & - & - & - & & 12 & 100,0 \\
\hline Médico (n=13) & 04 & 30,8 & 05 & 38,4 & 04 & 30,8 \\
\hline Auxiliar /Técnico de enfermagem $(n=75)$ & 14 & 19,0 & 17 & 22,0 & 44 & 59,0 \\
\hline Técnico de farmácia $(\mathrm{n}=12)$ & - & - & 02 & 17,0 & 10 & 83,0 \\
\hline Médico residente (n=2) & 01 & 50,0 & 01 & 50,0 & - & - \\
\hline Residente multiprofissional $(\mathrm{n}=11)$ & 07 & 63,6 & - & - & 04 & 36,4 \\
\hline Total $(n=157)$ & 32 & 20,4 & 37 & 23,6 & 88 & 56,0 \\
\hline
\end{tabular}

Tabela 3. Desvio entre o conhecimento declarado e o real demonstrado, sobre a lista de medicamentos potencialmente perigosos, pelos profissionais participantes da pesquisa sobre conhecimento de medicamentos potencialmente perigosos em uma maternidade de referência do nordeste Brasileiro, Brasil, 2016.

\begin{tabular}{|c|c|c|c|c|c|c|c|}
\hline \multicolumn{2}{|c|}{ Área de atuação/formação } & \multicolumn{5}{|c|}{$\begin{array}{l}\text { Desvio entre o conhecimento declarado e o real dos MPP da lista da } \\
\text { instituição } \\
\end{array}$} & \multirow[t]{2}{*}{ Total } \\
\hline & & -2 & -1 & 0 & 1 & 2 & \\
\hline \multirow[t]{2}{*}{ Enfermagem $^{1}$} & Valor absoluto & 49 & 17 & 35 & 5 & 1 & 107 \\
\hline & $\%$ do grupo & $45,8 \%$ & $15,9 \%$ & $32,7 \%$ & $4,7 \%$ & $0,9 \%$ & $100,0 \%$ \\
\hline \multirow[t]{2}{*}{ Farmácia ${ }^{2}$} & Valor absoluto & 0 & 6 & 16 & 2 & 0 & 24 \\
\hline & $\%$ do grupo & $0,0 \%$ & $25,0 \%$ & $66,7 \%$ & $8,3 \%$ & $0,0 \%$ & $100,0 \%$ \\
\hline \multirow[t]{2}{*}{ Médica $^{3}$} & Valor absoluto & 5 & 3 & 5 & 1 & 1 & 15 \\
\hline & $\%$ do grupo & $33,3 \%$ & $20,0 \%$ & $33,3 \%$ & $6,7 \%$ & $6,7 \%$ & $100,0 \%$ \\
\hline \multirow[t]{2}{*}{ Outra $^{4}$} & Valor absoluto & 0 & 2 & 8 & 1 & 0 & 11 \\
\hline & $\%$ do grupo & $0,0 \%$ & $18,2 \%$ & $72,7 \%$ & $9,1 \%$ & $0,0 \%$ & $100,0 \%$ \\
\hline \multirow[t]{2}{*}{ Total } & Valor absoluto & 54 & 28 & 64 & 9 & 2 & 157 \\
\hline & $\%$ do total & $34,4 \%$ & $17,8 \%$ & $40,8 \%$ & $5,7 \%$ & $1,3 \%$ & $100,0 \%$ \\
\hline
\end{tabular}

Nota: 1. Enfermeiros, auxiliares e técnicos de enfermagem; 2. Farmacêuticos e técnicos de farmácia; 3. Médicos e residentes médicos; 4. Residentes multiprofissionais; 5. Conhecimento declarado (avalia a extensão do conhecimento sobre os processos que envolvem os MPP) e conhecimento real da lista de MPP da instituição (representa o percentual de acerto do participante, em identificar os MPP em uma lista contendo MPP e outros medicamentos); 6. Teste Qui-quadrado; $\mathrm{p}=0,002 ;-2$, afirmou que o conhecimento estava desenvolvido, quando na verdade estava escasso, -1 afirmou que o conhecimento estava "desenvolvido" mas foi avaliado como "em desenvolvimento", 0 afirmou conhecimento correspondente com o avaliado, 1 afirmou que o conhecimento estava em "desenvolvimento" mas foi avaliado como "desenvolvido", 2 afirmou que estava "em desenvolvimento" mas foi avaliado como "escasso". 
Gráfico 1. Frequência de identificação de MPP pelos profissionais de saúde em maternidade do nordeste Brasileiro, Brasil, 2016.



Dentre os participantes do estudo, 27,4\% (n=43) destes relataram já ter presenciado a ocorrência de algum tipo de erro de medicação envolvendo MPP, exceto por médicos residentes. O relato de ter presenciado erros envolvendo MPP foi mais frequente entre os profissionais com maior tempo de serviço (Qui-quadrado, $\mathrm{p}=0,006$ ).

O questionário utilizado na pesquisa trazia uma questão aberta, permitindo o registro de sugestões e dúvidas sobre MPP. Este espaço foi utilizado por $57,3 \% \quad(n=90)$ dos participantes, sendo recebidas contribuições de todas as categorias profissionais, exceto a dos médicos residentes. As sugestões foram sumarizadas nos seguintes tópicos: instituir treinamento, disponibilizar a lista atualizada de MPP nas unidades; melhorar a estrutura para a distribuição e preparo de MPP; aumentar a participação dos farmacêuticos em visitas e treinamentos; divulgar a lista de MPP da instituição; enviar os MPP separados dos demais medicamentos para as unidades e realizar um maior número de treinamentos à noite.

A fase II do estudo foi realizada nos meses de outubro e novembro de 2016. Participaram do treinamento 164 profissionais, dentre eles 90 participantes da Fase I (57,3\% dos participantes desta fase) e os outros 74 participantes foram profissionais que não participaram da primeira fase da pesquisa, mas quiseram participar do treinamento. Durante o treinamento foi entregue aos participantes que quiseram particpar da capacitação um folder educativo sobre os medicamentos potencialmente perigosos abordando os seguintes assuntos: o que são MPP, quais MPP estão padronizados na instituição, como identificar um MPP na instituição, cuidados na prescrição, dispensação e administração. A capacitação abordou conceitos, lista de
MPP da instituição e o ciclo de utilização dos MPP, além de sanar as principais dúvidas identificadas.

\section{DISCUSSÃO}

Estudos de avaliação de conhecimento sobre MPP entre membros de equipes médicas, farmacêutica e de enfermagem são escassos, ${ }^{17,18}$ porém, estratégicos para mapear lacunas de segurança a serem preenchidas no processo assistencial. ${ }^{5,8,10-13,19,20}$

O perfil profissional dos participantes revelou a presença marcante de mulheres e a faixa etária prevalente de 31 a 39 anos, sendo semelhante ao identificado em diferentes estudos de perfil profissional no Brasil. ${ }^{21-23}$ Este perfil pode ter sido influenciado pela característica da instituição e pela maioria dos participantes pertencer à categoria da enfermagem que é, em grande parte, feminina. A predominância do sexo masculino somente foi verificada entre os médicos, refletindo dados da demografia médica no Brasil. ${ }^{23}$ Outra questão identificada no perfil é que a maioria dos profissionais, apesar de pouco tempo de trabalho na instituição (menos de um ano), era experientes (6 a 20 anos de profissão).

Quando analisadas as categorias profissionais de enfermeiros, farmacêuticos e médicos verifica-se que a maioria possuía pós-graduação, refletindo dados disponíveis na literatura, ${ }^{21-23}$ levando à reflexão sobre as lacunas de conhecimento prático na área de segurança do paciente, em especial sobre MPP, mesmo entre profissionais da saúde pós-graduados.

A capacitação profissional é reconhecida como um importante fator contribuinte para a redução dos erros e influencia 
positivamente a segurança do paciente..$^{5,10,11,13}$ No presente estudo foi observado um elevado percentual de pessoas não capacitadas sobre MPP nos últimos seis meses. Situação semelhante foi evidenciada por Lu e colaboradores em uma pesquisa randomizada realizada com 232 enfermeiros em Taiwan, na qual foi identificado que $74,1 \%$ dos participantes não tinham sido treinados sobre MPP $;^{10}$ e em estudo realizado por Hsaio e colaboradores, no qual foi observado que apenas $16,7 \%$ dos participantes tinham treinamento sobre MPP e $84,6 \%$ afirmaram a necessidade de um maior volume de treinamentos sobre o tema. ${ }^{11}$

No Brasil, estudo realizado no Hospital das Clínicas de São Paulo identificou de forma semelhante a outros estudos, ${ }^{11-12}$ reduzido percentual de enfermeiros $(26,7 \%)$ que haviam participado de treinamento sobre MPP, ${ }^{13}$ sendo a implementação de programa de capacitação em hospitais, abordando o tema MPP, uma importante estratégia para minimizar as falhas, evitando que as mesmas cheguem ao paciente na forma de danos. ${ }^{5}$

No presente estudo, a maioria dos profissionais não conhece todos os itens da lista de MPP da instituição, sendo imperioso que a referida lista seja disponibilizada nos postos de trabalho e divulgada aos profissionais para reduzir o risco de evento adverso envolvendo esses medicamentos. ${ }^{5,8}$

Quando analisado o conhecimento sobre a lista de MPP da instituição e o conhecimento geral declarado sobre MPP, foi identificada diferença estatisticamente significativa entre as categorias profissionais, revelando fragilidade de conhecimento sobre MPP, sobretudo entre médicos residentes, residentes multiprofissionais, médicos, técnicos de enfermagem e enfermeiros. Estes achados ratificam a necessidade de reduzir essa distorção de conhecimento entre os profissionais da instituição no ciclo de utilização do MPP, para promoção de maior segurança na assistência ao paciente, visto que os erros ocorrerão com maior frequência, no ponto frágil da cadeia assistencial, e a minimização do risco de eventos adversos somente é possível quando a equipe, como um todo, se responsabiliza pela prevenção destes eventos. ${ }^{19}$ Engels e Ciarkowski sugerem que o reduzido conhecimento dos médicos sobre o ciclo de uso do MPP na instituição, resulta do baixo envolvimento dos mesmos com o processo de administração, dispensação e estocagem dos MPP. ${ }^{17}$

Diferenças no conhecimento entre os profissionais de saúde sobre MPP foram também identificadas em estudo realizado por Engels e Ciarkowski ${ }^{17}$ e Tang e colaboradores. ${ }^{18}$ Para Engels e Ciarkowski, a educação profissional sobre o tema, contribui para que os profissionais aceitem e reconheçam seu papel e responsabilidades no processo de utilização de MPP; e para eliminar lacunas nas estratégias de redução do risco de utilização destes medicamentos, e que o conhecimento sobre MPP deve ser abordado na formação profissional e reforçado no trabalho. ${ }^{12,17}$

O desconhecimento dos residentes médicos e multiprofissionais sobre os MPP e o ciclo de utilização dos mesmos no hospital é, possivelmente, reflexo de lacuna na formação, na graduação dos profissionais de saúde, sobre segurança do paciente, e de ausência de experiência profissional anterior à residência. ${ }^{19}$ No Brasil, a Portaria do Ministério da Saúde $n^{\circ} 529$ aponta a necessidade da promoção de ações que impactem sobre a segurança do paciente nas instituições de saúde e prevê a inserção deste tema no ensino técnico, no nível superior e na pós-graduação das áreas de saúde, favorecendo o aprendizado não só dos residentes, mas de todos os profissionais envolvidos com a segurança do paciente..$^{20}$

Os MPP mais reconhecidos pelos participantes foram itens de consumo frequente na instituição. $\mathrm{O}$ cloreto de potássio endovenoso, a dobutamina e o sulfato de magnésio, são fármacos que podem ocasionar danos ao paciente, quando inadequadamente utilizados, requerendo educação para seu manejo, visando uma assistência segura. .,6,8,11 $^{-}$

A implementação de práticas de segurança no uso de MPP representa uma importante estratégia em direção à melhora na segurança do paciente..$^{5,8} \mathrm{~A}$ definição de protocolos; a disponibilização de manuais com doses máximas dos medicamentos; a elaboração e divulgação da lista dos MPP utilizados na instituição; a correta identificação, armazenamento em local seguro e de difícil acesso e realização de duplo-check na dispensação, preparo e administração dos MPP são consideradas estratégias relevantes e capazes de minimizar a ocorrência de erros quando aplicadas..$^{5,8}$

A pesquisa realizada por Engels e Ciarkowski envolvendo médicos, enfermeiros e farmacêuticos, identificou que os tipos de erros de medicação envolvendo MPP que os profissionais haviam mais presenciado estavam relacionados com a administração, dispensação, preparo e o armazenamento do MPP, e que a maioria deles não chegou ao paciente, porém, dois resultaram em morte. ${ }^{17}$ Hsaio e colaboradores identificaram que os erros com MPP, na administração, ocorreram por medicamento errado ou dose errada. ${ }^{11}$ Esses achados reforçam a importância da capacitação dos profissionais sobre o ciclo de utilização dos MPP.

$\mathrm{Na}$ segunda fase do estudo, sua realização foi dificultada pela recusa de parte dos profissionais em participar do treinamento, mesmo sendo informado que demandaria somente 15 minutos do seu tempo. Tal recusa se deu principalmente com os médicos e médicos residentes. Acredita-se que a recusa tenha ocorrido em virtude da baixa percepção do papel desempenhado pelo médico no ciclo de utilização de MPP e também pela baixa percepção do impacto dos erros envolvendo MPP sobre a qualidade da assistência e segurança do paciente. Essa baixa percepção também foi relatada por Engels e Ciarkowski. ${ }^{17}$

Os resultados do presente estudo podem ter sido influenciados pela dificuldade de entendimento, de alguns participantes, sobre como responder as questões que utilizavam a escala de Likert. Outra limitação foi a dificuldade em captar médicos e residentes médicos para responder ao questionário da Fase I e para participar dos treinamentos na Fase II. 
Foi evidenciada na Fase I fragilidade no conhecimento sobre MPP entre profissionais de saúde. Isto se deve à não realização de capacitações na instituição e possivelmente influenciada pela não abordagem do tema durante a graduação e cursos técnicos, conforme determina legislação brasileira vigente.

Intervenções educativas foram feitas na Fase II com o intuito de diminuir a fragilidade identificada nos profissionais que fazem parte do ciclo de utilização de MPP na instituição, sensibilizando-os para as consequências danosas dos erros envolvendo estes fármacos. Na Fase II houve 164 participantes,

\section{REFERÊNCIAS}

1. World Health Organization. World Alliance for Patient Safety. Global Patient Safety Challenge: 2005-2006. Genova: WHO; 2005 [acesso em: 02 mar 2018]. Disponível em: http://www.who.int/ patientsafety/events/05/GPSC Launch ENGLISH FINAL.pdf

2. Runciman W, Hibbert P, Thomson R, Van Der SchaafT, Sherman H, Lewalle P. Towards an International Classification for Patient Safety: key concepts and terms. Int J Qual Health Care. 2009;21(1):18-26.

3. Brasil. Ministério da Saúde. Protocolo de segurança na prescrição, uso e administração de medicamentos. Brasília: Ministério da Saúde; 2013 [acesso em: 02 mar 2018]. Disponível em: https://www20.anvisa.gov.br/segurancadopaciente/index.php/ publicacoes/item/seguranca-na-prescricao-uso-e-administracao-demedicamentos

4. Graham S, Clopp MP, Kostek NE, Crawford B. Implementation of a High-Alert medication program. Perm J. 2008;12(2):15-21.

5. Cohen MR, Smetzer JL, Tuohy NR, Kilo CM. High-alert medications: safeguarding against errors. In: Cohen MR, editor. Medication Errors. 2nd ed. Washington (DC): American Pharmaceutical Association; 2007. p. 317-411.

6. Rosa MB, Perini E, Anacleto TA, Neiva HM, Bogutchi T. Erros na prescrição hospitalar de medicamentos potencialmente perigosos. Rev Saúde Pública. 2009;43(3):490-8.

7. Instituto para práticas seguras no uso de medicamentos. Medicamentos potencialmente perigosos de uso hospitalar e ambulatorial - Listas atualizadas 2015. Boletim ISMP Brasil [Internet]. 2015 [acesso em: 27 jan 2016];4(3):1-8. Disponível em: http://www. ismp-brasil.org/site/wp-content/uploads/2015/12/V4N3.pdf

8. Ministerio de Sanidad y Consumo, Instituto para el Uso Seguro de Medicamentos. Universidad de Salamanca. Practicas para mejorar la seguridad de los medicamentos de alto riesgo [Internet]. 2007 [ acesso em: 27 jan 2016]. Disponível em: http://www.ismp-espana. org/ficheros/Practicas\%20para\%20mejorar\%20la\%20seguridad\%20 de $\% 2010$ \% $\% 20$ medicamentos $\% 20$ de $\% 20$ alto $\% 20$ riesgo..pdf

9. Bohomol E. Erros de medicação: estudo descritivo das classes dos medicamentos e medicamentos de alta vigilância. Esc Anna Nery. 2014;18(2):311-16.

10. Lu MC, YU S, Chen IJ, Wang KW, Wu HF, Tang FI. Nurses' knowledge of high-alert medications: a randomized controlled trial. Nurse Educ Today. 2013;33(1):24-30. desses, 74 profissionais de saúde mesmo não tendo respondido o questionário quiseram participar da capacitação. A capacitação abordou conceitos, lista de MPP da instituição e o ciclo de utilização dos MPP, além de sanar as principais dúvidas identificadas na questão aberta do questionário respondido pelos participantes da Fase I. Novas estratégias devem ser feitas para sanar a deficiência identificada entre os profissionais de saúde, como implementar capacitações em serviço sobre o tema, e divulgação e disponibilização de protocolos e manuais com doses máximas dos MPP utilizados na instituição.

11. Hsaio GY, Chen IJ, Yu S, Wei IL, Fang YY, Tang FI. Nurses' knowledge of high-alert medications: instrument development and validation. J Adv Nurs. 2010;66(1):177-90.

12. Lo TF, YU S, Chen IJ, Wang KW, Tang FI. Faculties' and nurses' perspectives regarding knowledge of high-alert medications. Nurse Educ Today. 2013;33(3):214-21

13. ZanettiAC. Segurança do paciente e medicamentos potencialmente perigosos: adaptação transcultural de um questionário [dissertação]. Ribeirão Preto: Escola de Enfermagem de Ribeirão Preto da Universidade de São Paulo; 2014 [acesso em: 03 mar 2018]. 136 p. Disponível em: http://www.teses.usp.br/teses/disponiveis/22/22132/ tde-30032015-181311/pt-br.php

14. Santos GE . Cálculo amostral: calculadora on-line [Internet]. [acesso em: 30 jan 2017]. Disponível em: http://www.calculoamostral. vai.la

15. Likert RA. Technique for the measurement of attitudes. Archives in Psychology. 1932;140:1-55.

16. Moreira MA, Masini EA. Aprendizagem significativa: a teoria de David Ausubel. São Paulo: Editora Moraes;1982.

17. Engels MJ, Ciarkowski SL. Nursing, pharmacy and prescriber knowledge and perceptions of high-alert medications in a large, academic medical hospital. Hosp Pharm. 2015;50(4):287-95.

18. Tang SF, Wang X, Zhang Y, Hou J, Ji L, Wang ML, et al. Analysis of high alert medication knowledge of medical staff in Tianjin: a convenient sampling survey in China. J Huazhong Univ Sci Technolog Med Sci. 2015;35(2):176-82.

19. Colet CF, Holzle DEM, Seidler RE, et al. Conhecimento aos profissionais de saúde sobre o uso de varfarina em ambiente hospitalar. Rev Soc Bras Clin Med., 2016, 14(4):204-11.

20. Brasil. Ministério da saúde. Portaria $n^{\circ} 529$, de $1^{\circ}$ de abril de 2013. Institui o Programa Nacional de Segurança do Paciente (PNSP) [Internet]. Brasília; 2013 [acesso em: 21 mar 2018]. Disponível em: http://bvsms.saude.gov.br/bvs/saudelegis/gm/2013/ prt0529_01_04_2013.html

21. Machado MH, Aguiar W Filho, Lacerda WF, Oliveira E, Lemos $\mathrm{W}$, Wermelinger $\mathrm{M}$, et al. Características gerais da enfermagem: Perfil sócio demográfico. Enferm Foco. 2015;6(1/4):11-17. 
22. Serafin C, Correia D Júnior, Vargas M. Perfil do farmacêutico no Brasil [Internet]. Brasília: Conselho Federal de Farmácia; 2015 [acesso em: 02 mar 2018]. Disponível em: http://www.cff.org.br/ userfiles/file/Perfil $\% 20$ do $\% 20$ farmac $\%$ C3\%AAutico $\% 20$ no $\% 20$ Brasil\%20_web.pdf
23. Scheffer M, Cassenote A, Biancarelli A. Demografia médica no Brasil 2015 [Internetr]. São Paulo: Departamento de Medicina Preventiva, Faculdade de Medicina da USP; Conselho Regional de Medicina do Estado de São Paulo; Conselho Federal de Medicina; 2015 [acesso em: 02 mar 2018]. 284 p. Disponível em: http://www. usp.br/agen/wp-content/uploads/DemografiaMedica30nov2015.pdf

\section{Como citar:}

Souza AA, Néri ED, Gomes GC, Lopes EM, Fonteles MM, Meira AS, et al. Avaliação do autoconhecimento da equipe médica, de enfermagem e farmácia sobre medicamentos potencialmente perigosos. Rev Med UFC. 2019 jan-mar;59(1):21-29. 